\title{
Letter to the Editor: Are American Surgical Residents Prepared for Humanitarian Deployment? A Comparative Analysis of Resident and Humanitarian Case Logs
}

\author{
David J. Read ${ }^{1}$
}

Published online: 25 April 2018

(C) Société Internationale de Chirurgie 2018

Dr. Lin et al. [1] should be congratulated for their thorough comparison of operative experience of US surgical residents during training versus that of Médecins Sans Frontières (MSF) deployed surgeons. Such an analysis is a useful contribution in defining the professional and training requirements likely required for a surgeon wishing to deploy on a variety of humanitarian missions. However, the conclusion that US trained residents are unprepared for humanitarian deployment is overstated, as it only considers one model of care, that of the solitary pluripotent surgeon who can practise across a broad area of expertise. Unquestionably, this model of care has served several humanitarian actors well over many decades and has definite financial and logistic advantages. It is agreed that the increasing drive towards subspecialisation will make such surgeons harder to find, but an awakening of the requirements of global surgery may offset this somewhat.

Australian Medical Assistant Teams (AUSMAT) have a differing model of care, based upon the ethos that as much as practical, a deployed surgeons scope of practice abroad should align to their practice at home, and that the recipient nation's patients deserve a level of care at least equivalent to what is, or was, available locally. This has led to the model of a three person surgical team consisting of an obstetrician/gynaecologist, an orthopaedic surgeon and general surgeon, which can be varied according the requirement for the mission. For example, in the wake of Typhoon Haiyan in 2013, the AUSMAT Emergency Medical Team Type 2 offered general and orthopaedic surgery, whilst the host nation continued to provide

David J. Read

mrdavidread@internode.on.net

1 National Critical Care and Trauma Response Centre, Dawin, Australia obstetric services [2]. There are numerous other examples of multi-surgeon teams deploying in the humanitarian setting.

The presented data would support that US trained resident's caseload roughly aligns with MSF's pooled general surgical caseload, and hence they may indeed be appropriate candidates to deploy as part of a multi-surgeon team. Austere environment training courses are offered as a solution, but are no substitute for the clinical experience gained by completing specialty training. Such courses are best equipped to teach already competent surgeons to adapt their techniques to the proclivities of the austere environment. Humanitarian actors need to decide for themselves how they will provide surgical services in the future, by either training increasingly subspecialised surgeons to work under a single surgeon model, or to consider other models that align more with modern surgical practice. Both models have differing resource implications, strengths, weaknesses and applicability, and neither is perfect. There is room enough in this space for humanitarian surgeons trained under both models.

\section{References}

1. Lin Y, Dahm J, Kuschner A et al (2018) Are American surgical residents prepared for humanitarian deployment? A comparative analysis of resident and humanitarian case logs. World J Surg 42:32-39. https://doi.org/10.1007/s00268-017-4137-x

2. Read D, Holian A, Poutawera V et al (2016) Surgical workload of a foreign medical team after Typhoon Haiyan: surgery after Typhoon Haiyan. ANZ J Surg 86(5):361-365 